\title{
PERFORMANCE ANALYSIS OF PF MAC SCHEDULER UNDER VARYING FEEDBACK DELAY IN LTE AND LTE-A EUTRAN
}

\author{
Ankit Saxena* and Ravi Sindal*
}

\begin{abstract}
Evolved Universal Mobile Telecommunications System (UMTS) Terrestrial Radio Access Network (EUTRAN) deals with high uplink and downlink throughput. The Proportional Fair (PF) Medium Access Control (MAC) scheduler offered a good balance between allocated resources and fairness among the User Equipment (UE) in comparison with other schedulers. In the PF scheduler, at each cycle of resource allocation, a feedback mechanism ensures that operational resource requirements are met while maintaining a degree of fairness among the UE. We analyze the effect of feedback Transmission Time Interval (TTI) delay on two typical EUTRAN networks, in terms of Key Performance Indicators (KPIs). One network is referred to as EUTRAN LTE and the other as EUTRAN LTE-A, which fulfils high throughput requirement. We evaluate the performance of the PF MAC scheduler for both networks under varying feedback delay in TTI. Simulated results show that the delay in TTI affects the performance of resource allocation in terms of throughput, fairness, and spectral efficiency. Feedback delay improves network performance by compensating effect of imperfect channel condition.
\end{abstract}

\section{Key Words}

Proportional Fair Scheduler, Long Term Evolution, Long Term Evolution-Advance

\section{Introduction}

Long Term Evolution (LTE), ${ }^{1}$ promoted as $4 \mathrm{G}$, is a wireless communication system providing high speed data for user equipment (UE). The standards are developed by 3rd Generation Partnership Project (3GPP) [1], under two mobile data transmission technologies, termed as LTE-Time

* Institute of Engineering and Technology Devi Ahilya University Indore, India; e-mail: \{asaxena2014, rsindal\}@ietdavv.edu.in, ankitsaxena_17@hotmail.com

${ }^{1}$ LTE/LTE-A" denotes "Long Term Evolution" and "LTEAdvance".

Recommended by Dr. Leone Monticone

(DOI: 10.2316/Journal.214.2016.4.214-1080)
Division Duplex (LTE-TDD) and LTE-Frequency Division Duplex (FDD), supported by end-to-end IP connectivity using bearer through LTE Evolved Packet Core (EPC) on radio part and packet data network $(\mathrm{PDN})$ on server part, forming an all-IP flat architecture of the system called as Evolved Packet System (EPS). The main features include peak download rates of $299.6 \mathrm{Mb} / \mathrm{s}$ and upload rates up to $75.4 \mathrm{Mb} / \mathrm{s}$ based on UE (with $4 \times 4$ antennas using $20 \mathrm{MHz}$ of spectrum, as a transmission mode simultaneous connection allowed).

In a mobile network, transmission resources are available in a pool, to meet the dynamic requirement of resources with mobility. Therefore, an optimum designing rule, that is a trade-off between availability and blocking, should be performed; supervision and control are based on an effective resource utilization factor. To make a costeffective network setup, it is always suggested to design a network that fulfils the peak load requirement with a maximum $60 \%-70 \%$ utilization for the transmission requirement and with less than $30 \%$ utilization for core element requirements such as the access nodes/switches. This dimension may vary according to dynamic customers' requirements. As non-usage of resources causes an increase in operational and maintenance costs, as well as upholding loss of power/energy, efficient resource utilization is a prime concern in enhancing green communication and reducing the carbon footprint. This idea can be supported by effective resource scheduling at the media access control (MAC).

In EUTRAN, LTE technology is also supported by multiple MAC schedulers. We verify the behavior of the available schedulers through simulation, and measure the performance in terms of Key Performance Indicators (KPIs). To offer high uplink and downlink throughput, the spectrum is divided into subcarriers (using OFDMA) and allocated to the UE. At high frequency, the most interesting task is to serve radio propagation losses, especially at the cell edges, where low signal-to-interference-noise ratio (SINR) gives relatively smaller coverage and capacity. To overcome the stated problem, the MAC scheduler allocates resources through the feedback mechanism by using 
different algorithms such as round robin (RR), proportional fair $(\mathrm{PF})$, best CQI, maximum TP, and resource fair (RF) [2], to improve resource efficiency, thereby to satisfy the user's requirements and to achieve a definite degree of fairness. Through system level simulations, the performance of PF scheduler is evaluated and compared for LTE and LTE-A EUTRAN network in terms of KPI for cell and UE.

\subsection{Related Works}

Authors in [3] discuss a nonlinear regression function based on the prediction of the Channel Quality Indicator (CQI) from previous feedback with the PF LTE scheduler, and their simulation results show that prediction function improves in terms of throughput. Authors in [4] discuss challenges in scheduling user transmissions for the downlink of an LTE network; they propose PF and suboptimal PF scheduler and measure performance in terms of throughput and SINR. Authors in [2] describe the challenges of multiuser scheduling in the downlink of 3GPP and LTE networks. They also propose CQI feedback based on a linearized model using mutual information effective SNR mapping; they calculate the average CQI per UE resources and compare performance of the scheduler in terms of fairness and throughput. Authors in [5] using system level simulations compare the performance of five different schedulers under uniform LTE (EUTRAN) network in terms of KPIs for cell and UE.

Authors in [6] discuss the measurement and comparison of LTE downlink transmission performance (SISO) and the transmission diversity mechanisms with RR and PF scheduler. They have concluded that RR offers the best fairness for the UE, and that PF can maintain a better balance between fairness and throughput. Authors in [7] explore on PF scheduling and demonstrate system performance in terms of throughput and fairness.

Authors in [8] discuss performance improvement for the PF scheduler by proposing a partitioning algorithm of resources between nodes and the relay node, and conclude with an improved fair degree of resource distribution among UE, the efficient utilization of resources, and system throughput. Authors in [9] describe an analysis of cell re-selection and conclude that in EUTRAN, selected cells have the highest $\mathrm{RSRP}^{2}$ and the selected delay varies with the mobility of UE.

Authors in [10] explore basic concepts of a downlink packet scheduling strategy in LTE cellular networks. Their paper includes a survey of different schemes to optimize the trade-off between spectral efficiency and fairness, along with other key design issues. Authors in [11] show a significant improvement of CoMP in comparison with noncoordinated (N-CoMP) link adaptation in LTE-A, with interference.

Authors in [12] describe work on end-to-end packet delay in a two-hop relay network that consists of two groups of wireless nodes, in LTE/LTE-A systems. They recommend evaluating other performance metrics, such as throughput,

\footnotetext{
${ }^{2}$ RSRP stands for "Reference Signal Received Power".
}

for the two-hop relay network. In [13], the authors investigate a cooperative Radio Access Network (RAN) wide MAC layer protocol based on a random network coding using finite state Markov Chain channel model over link-level simulator. Authors have also highlighted the simplicity and flexibility required for future LTE-A RANs.

Authors in [14] propose an algorithm to solve the bandwidth requirement between a baseband unit and a remote radio unit by removing redundancy in the LTE spectral domain, using a low-latency baseband signal compression technique. Further, the authors focus on resource optimization of a Common Public Radio Interface (CPRI), using redundant spectrum bandwidth and compressing the bit width. As a result, the authors show that data loss is negligible at low compression ratio, and as a future scope of work the authors propose to identify a method of compression to optimize performance and resource consumption on CPRI interface. Authors in [15] evaluate the performance of three basic packet schedulers at downlink such as: RR, maximum rate, and proportional algorithms, for a $3 \mathrm{GPP}$ LTE cellular network by executing a simulation in different channel conditions in terms of maximum throughput and fairness metrics.

\subsection{Major Contributions of this Work}

1. Different KPIs to measure the performance of the network, such as cell throughput, UE throughput, fairness of resource allocation, resource availability or blocking, and UE spectral efficiency can be identified.

2. Cell peak throughput can be improved by 5-6 TTI feedback delay in an LTE EUTRAN and 1-3 TTI feedback delay in LTE-A.

3. Balanced throughput in terms of fairness can be achieved by 2-3 TTI feedback delay in LTE and $4-6$ TTI feedback delay in LTE-A.

4. Spectral efficiency and UE throughput can be improved through 2-6 TTI feedback delay in both LTE and LTE-A.

\subsection{Terminology}

In this work, the terms Relay Node (RN) and Remote Radio Head (RRH $)^{3}$ collaboration refer to systems where eNodeB (eNB) interchange received signals or information connected to certain UEs to increase data rates. The performance of the network is measured through different KPIs, for example UE throughput, spectral efficiency, and fairness index. Similarly, the following KPIs, CQI, Bit Error Rate (BER), and Block Error Rate (BLER), are used to measure performance of the Transmitting Point (TP).

\subsection{Outline}

Section 2 describes problems that arise in resource allocation in EUTRAN with LTE and LTE-A. Section 3 hosts

\footnotetext{
${ }^{3}$ RRH acronym "Remote Radio Head" is same as relay node.
} 


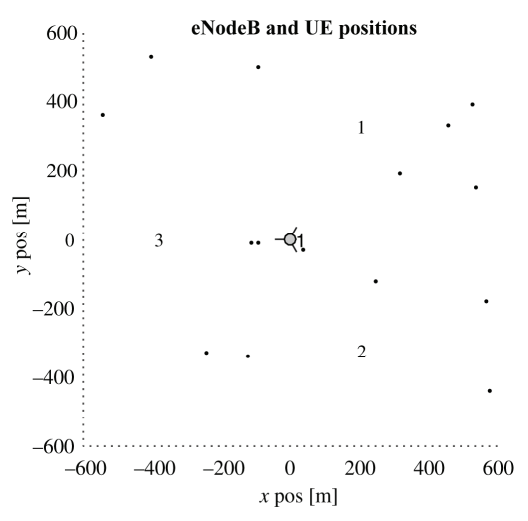

Figure 1. Graphical presentation of eNB with UE. The region is divided into three different sectors $1-3$.

the working principle of PF; Section 4 describes an experimental setup with simulator and their comparative performance analysis with respect to the achievable throughput. This work is concluded in the last section.

\section{Problem Description in EUTRAN}

In this section, we give a brief introduction to the optimization problem in EUTRAN for LTE and LTE-A, in terms of efficient resource utilization. As the resources for network design are available in a pool referred to as redundant or alternative resources, here we find a trade-off condition between the additional resources offer that increases the operating costs and promotes efficient utilization of resources.

\subsection{System Model - LTE Network}

When we have an LTE-A EUTRAN network, the system model comprises of eNB and UE with auxiliary supporting units such as an antenna for both transceivers. Figure 1 shows the basic LTE system modelled with a single eNB and UE. Here the UE gets connected with the dominating radio signal from the corresponding eNB sector. Each eNB has three sectors connected with a separate antenna assembly. They are called sectors $1-3$.

\subsection{System Model - LTE-A}

In an LTE-A EUTRAN network, the system model comprises of eNB and UE with auxiliary supporting units such as an antenna for both transceivers. Figure 2 exhibits a basic LTE system modelled with a multi eNB with a ring of RN or RRHs and UEs. Here the UE gets connected with a dominating radio signal from the corresponding eNB or RN. Each eNB is surrounded by RN or RRHs with a separate antenna supporting each assembly. The basic advantage of deploying a RRH is to provide high power and a quality radio signal that enables the UE to communicate at a high throughput rate.

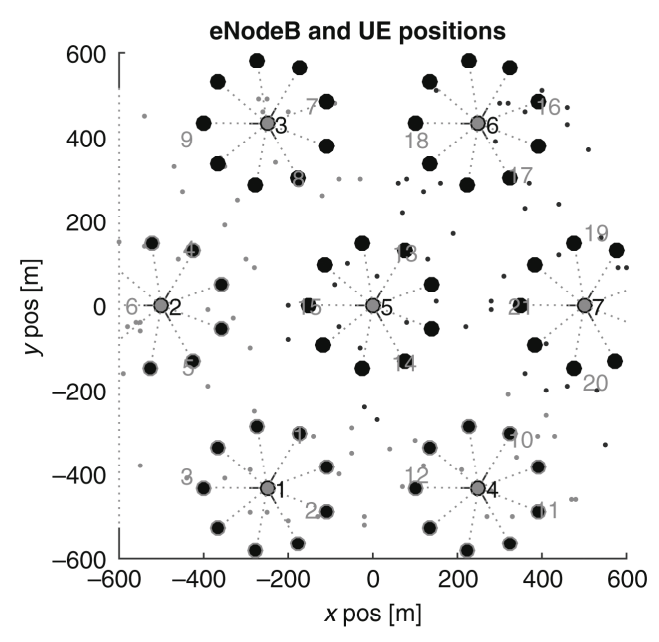

Figure 2. Graphical presentation of multiple eNB with UE, and ring relay/remote nodes.

\subsection{Key Performance Indicator}

To measure system performance, different counters and stats are defined. They are:

1. Cell and UE throughput: This is referred to as traffic flow rate in terms of data measured in bits per second; it can be calculated on cell and UE level. On cell level it is an aggregation of all UE belonging to a particular cell, while on UE level it refers to a specific UE.

2. UE spectral efficiency or bandwidth efficiency: This is referred to as information rate that can be transmitted over given bandwidth for a specific communication and limited to physical layer utilization and channel access control (MAC). Information rate is measured in bit/symbol or bit/channel (bpcu).

3. Fairness index: To measure and maintain resource distribution among UE, Jain fairness index is preferred as it helps to identify the quantitative distribution of resources among users [16]:

$$
J\left(x_{1}, x_{1}, \ldots, x_{n}\right)=\frac{\left(\sum_{i=1}^{n} x_{i}\right)^{2}}{n \sum_{i=1}^{n} x_{i}^{2}}
$$

This is a reference parameter value of fairness where there are $n$ users. The value that corresponds to connect allocated in RB for each user is $x_{i}$. Actual results will vary from $1 / n$ (worst condition) to 1 (good condition). The best condition will be obtained when all the users receive the same amount of allocated RB.

4. CQI: It includes SINR and BLER. UE frequently shares CQI with a corresponding TP; resultant eNB allocates resource towards corresponding adaptive Modulation Coding Scheme for respective UE.

\section{Working of Proportional Fair Scheduler}

The PF algorithm performs the UE scheduling when its instantaneous channel quality is comparatively higher than its own average channel condition over time. Let $\varphi$ be the Modulation and Coding Scheme (MCS) index assigned to a user that depends on the corresponding CQI. Using the 


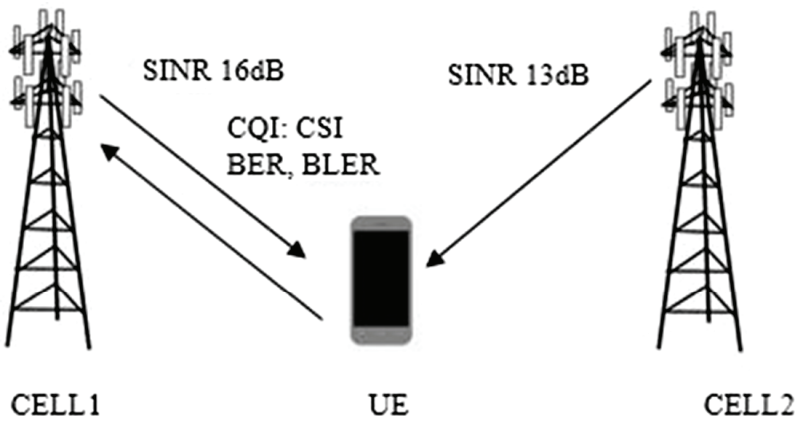

Figure 3. Illustration of cell in modern EUTRAN architecture.

Transport Block Size (TBS) mapping $S\left(\phi_{j}, \beta\right)$, the transport block size is defined by 3GPP standard TS36.213, where $\beta$ is the number of resource blocks used. This number is decided according to the bandwidth of a transmission channel. Let us assume $T_{j}(t)$ be the past average throughput of the user $j$. Hence, the rate achieved by the user $j$ is given by (2):

$$
\Psi_{j}(k, t)=\frac{S\left(\phi_{j}, \beta\right)}{\tau}
$$

where $\tau$ is the transmission time interval defined in (1) and index $\mathrm{i}_{k}(t)$. to be scheduled is defined by $(3)$, where $T_{j}(t)$. is the past throughput performance by $j$ th user:

$$
\mathrm{i}_{k}(t)=\operatorname{argmax}_{j=1, \ldots, N} \frac{\Psi_{j}(k, t)}{T_{j}(t)}
$$

The channel performance $T_{j}(t)$ is an exponential moving average defined by (4), where $\alpha$ is the time constant (in number of frames), and $\check{\mathrm{T}}_{j}(t)$ is the actual throughput achieved by the $j$ th user in sub-frame $t$ :

$$
T_{j}(t)=\left(1-\frac{1}{\alpha}\right) T_{j}(t-1)+\frac{1}{\alpha} \check{\mathrm{T}}_{j}(t)
$$

PF algorithms drive the allocation of resource based on channel imperfection. As the UE moves away from the eNB, the PF scheduler allocates less resources and provides reduced throughput due to a weaker radio link.

We experimentally evaluate the performance of the PF scheduler through two typical EUTRAN networks by varying feedback delay and comparing their results through KPIs. In LTE EUTRAN, a UE gets connected with corresponding eNB that has good SINR via the supporting antenna assembly. Although the UE is attached to the eNB, the UE periodically shares the CQI report declaring channel state information which includes BER, BLER, and SINR through a feedback mechanism to the corresponding eNB. Based on the CQI feedback report, the MAC $\mathrm{PF}$ scheduler allocates resources through the eNB to the corresponding UE as shown in Fig. 3.

In the same way as earlier, we have taken two cooperative transmission points in an LTE-A EUTRAN with RN/RRH networks: TP1 (eNB) and TP2 (RRH), each capable of transmitting the Physical Downlink Shared Channel (PDSCH) to the UE. TP1 is the serving cell for the UE. The network selects the PDSCH transmission point
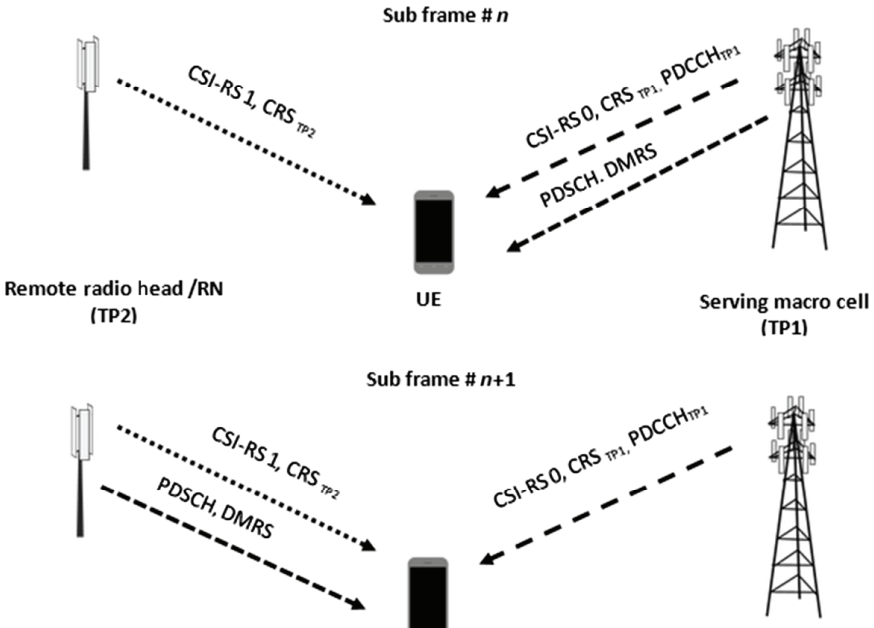

Remote radio head /RN

(TP2)

Sub frame \#n+1

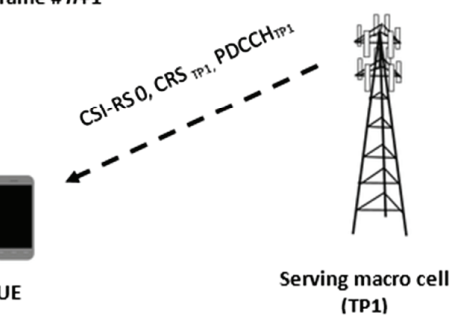

Figure 4. Illustration of a LTE-A network that consists of two TP $(\mathrm{eNB}=\mathrm{TP} 1$ and $\mathrm{RRH} / \mathrm{RN}=\mathrm{TP} 2)$.

Table 1

Simulation Configuration LTE EUTRAN

\begin{tabular}{|c|c|}
\hline Frequency $(\mathrm{Hz})$ & $2.14 \mathrm{E}+09$ \\
\hline Bandwidth $(\mathrm{Hz})$ & $20,000,000$ \\
\hline nTX & 2 \\
\hline nRX & 4 \\
\hline tx mode & 4 \\
\hline Simulation time (TTI) & 10 \\
\hline Inter eNodeB distance (m) & 500 \\
\hline nr eNodeB rings & 0 \\
\hline Minimum coupling loss (dB) & 70 \\
\hline Macroscopic path loss model & TS36942 \\
\hline eNodeB tx power $(\mathrm{dBm})$ & 46 \\
\hline Shadow fading type & None \\
\hline UE distribution & Constant UEs per cell \\
\hline UE per eNodeB & 5 \\
\hline UE speed $(\mathrm{m} / \mathrm{s})$ & 1.3889 \\
\hline Scheduler & Proportional fair \\
\hline Power allocation & Homogeneous \\
\hline Feedback channel delay (TTI) & $0-6$ \\
\hline Antenna Azimuth offset (degree) & 30 \\
\hline Output file name suffix & Tri-sector tilted \\
\hline
\end{tabular}

3 cells, 15 UEs; simulation length: 10 TTIs; scheduler: proportional fair; mode: $2 \times 4$, CLSM 


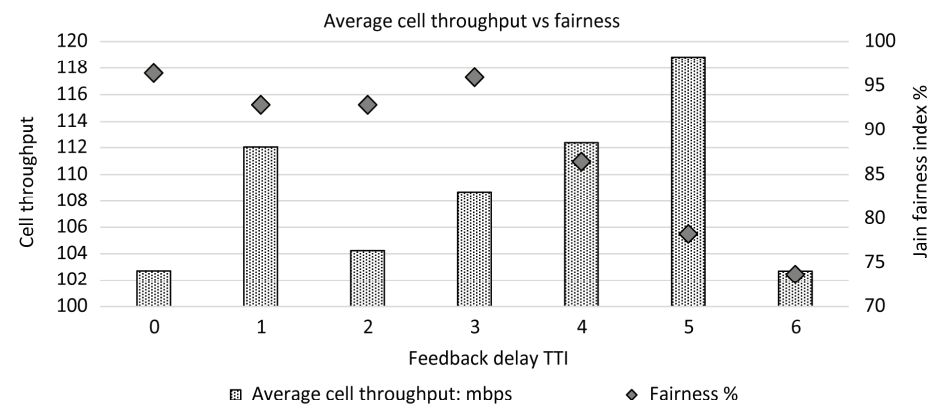

Figure 5. Performance analysis of PF scheduler in LTE EUTRAN without RN/RRH in terms of throughput and resource fairness index.

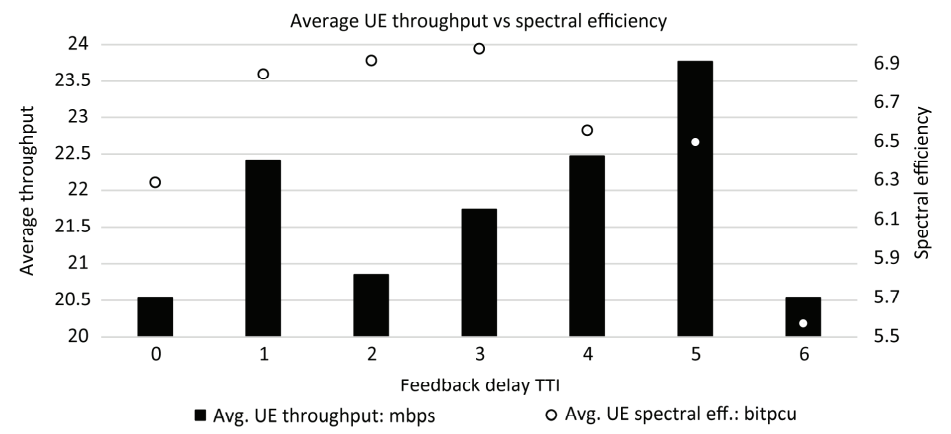

Figure 6. Performance analysis of PF scheduler in LTE EUTRAN without RN/RRH in terms of UE average throughput and UE average spectral efficiency with varying feedback delay.
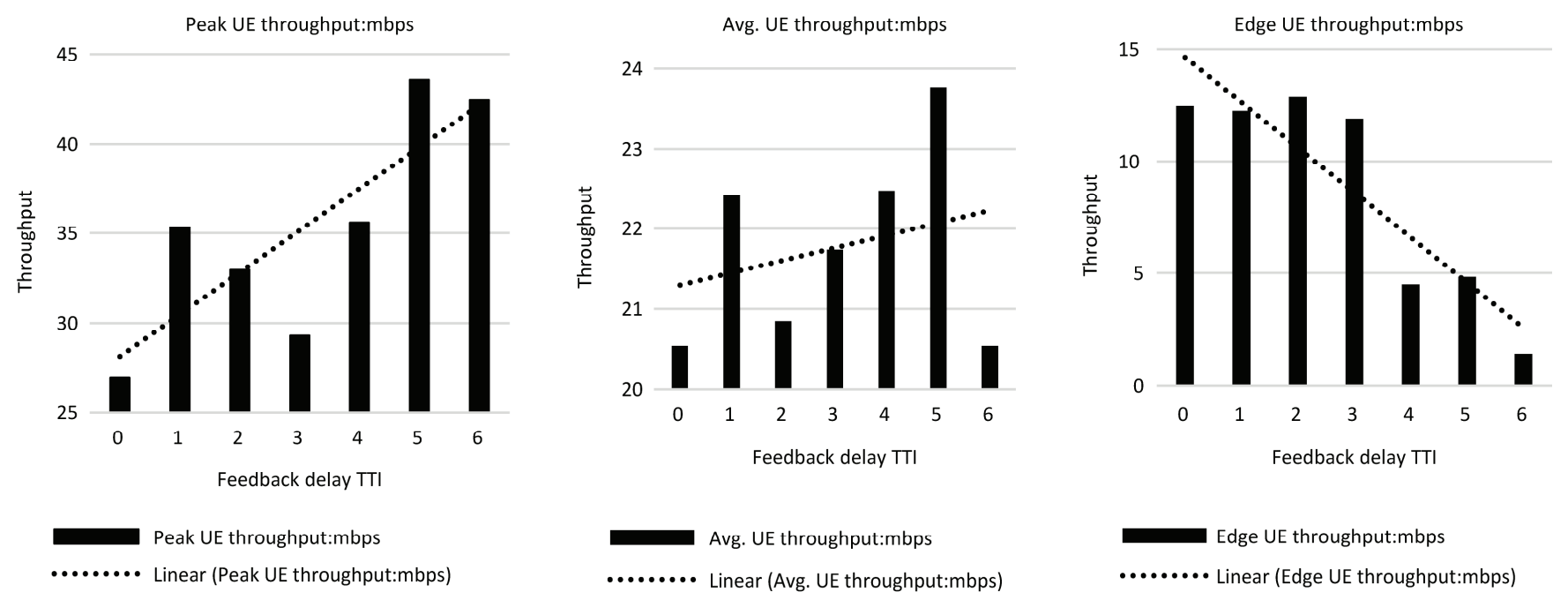

Figure 7. Throughput performance in typical PF scheduler EUTRAN network without RN/relay for UE at peak, average, and edge, under varying feedback delay.

and the modulation and coding scheme using the CQI) reported by the UE as shown in Fig. 4. Both points transmit a CSI-RS and Cell-specific Reference Signal (CRS). The serving cell also transmits downlink control information for the UE in the Physical Downlink Control Channel (PDCCH). The PDSCH transmission point can change each subframe to take advantage of instantaneous channel conditions. Here also, based on the CQI feedback report, the MAC PF scheduler allocates resources through the eNB to its corresponding UE.

\section{Experimental Setup and Result Analysis}

We establish EUTRAN networks with the help of a system level simulator in MATLAB ${ }^{\circledR}$ and measure KPI with the PF scheduler. Here we form two typical EUTRAN networks, LTE (as shown in Fig. 1) and LTE-A (as shown in Fig. 2), both being under varying feedback delay.

\subsection{LTE Network}

An LTE EUTRAN network consists of 3 cells and 15 UEs, with a MAC scheduler as PF with transmission mode of $2 \times 4$ Closed Loop Spatial Multiplexing (CLSM) for simulation time of 10 TTIs according to Table 1.

Figure 5 represents the results of LTE network simulation in terms of cell throughput rates and resource distribution among UEs in the network. As shown in the graph, 
we conclude that cell throughput increases at feedback delay from 1 to 5 TTI and then decreases. Fairness index also comes in the range of $90 \%-95 \%$ for $0-3$ TTI while it declines abruptly to below $85 \%$ from 4 TTI onwards.

Table 2

Simulation Configuration LTE-A EUTRAN

9 cells, 45 UEs; simulation length: 10 TTIs; scheduler: proportional fair; mode: $1 \times 4$, CLSM

\begin{tabular}{|c|c|}
\hline Frequency $(\mathrm{Hz})$ & $2.14 \mathrm{E}+09$ \\
\hline Bandwidth (Hz) & $20,000,000$ \\
\hline nTX & 1 \\
\hline $\mathrm{nRX}$ & 4 \\
\hline tx mode & 4 \\
\hline Simulation time (TTI) & 10 \\
\hline Network geometry & Regular hexagon \\
\hline Inter eNodeB distance (m) & 500 \\
\hline $\mathrm{nr}$ eNodeB rings & 1 \\
\hline Minimum coupling loss (dB) & 70 \\
\hline Macroscopic path loss model & TS36942 \\
\hline eNodeB tx power $(\mathrm{dBm})$ & 40 \\
\hline Shadow fading type & None \\
\hline UE distribution & Constant UEs per cell \\
\hline UE per eNodeB & 5 \\
\hline UE speed $(\mathrm{m} / \mathrm{s})$ & 1.3889 \\
\hline Scheduler & Proportional fair \\
\hline Power allocation & Homogeneous \\
\hline Feedback channel delay (TTI) & $0-6$ \\
\hline RRHs enabled & 1 \\
\hline Cyclic prefix & Normal \\
\hline
\end{tabular}

Figure 6 represents the results of the LTE network simulation in terms of average UE throughput rate and spectral efficiency of the network. As shown in the graph, we conclude that UE average throughput increases for feedback delay from 0 to 5 TTI and then decreases, and spectral efficiency also increases in the range of $6-7 \mathrm{bit} / \mathrm{cu}$ for $0-5$ TTI while it declines abruptly to below $6 \mathrm{bit} / \mathrm{cu}$ from 5 TTI onwards.

In Fig. 7, we draw UE throughput under a varying feedback delay in a typical PF Scheduler EUTRAN Network. Simulation configuration is defined as in Table 1. In the first graph, UE peak throughput increases with the feedback delay in PF scheduler. In the second graph, the UE average throughput also increases, but the slope is less than in the UE peak throughput. However, in the third graph, throughput decreases when feedback delay increases. For better understanding, we refer trend line to identify the behavior of UE throughput under three different aggregation levels.

\subsection{LTE-A Network}

An LTE-A EUTRAN network comprises of 9 cells and 45 UEs, with a MAC scheduler as $\mathrm{PF}$ with transmission mode of $1 \times 4$, CLSM for simulation time of 10 TTIs as in Table 2.

Figure 8 represents the result of LTE-A network simulation in terms of cell throughput rates and resource distribution among UEs in the network. As shown in the graph, we conclude that cell throughput keeps fixed in the range between 355 and $350 \mathrm{Mb} / \mathrm{s}$ for feedback delay from 1 to 6 TTI, and the fairness index initially decreases from $98 \%$ to $95 \%$ for a range of $0-3$ TTI while it increases abruptly above $99 \%$ from 4 TTI onwards.

Figure 9 represents the results of LTE-A network simulation in terms of the UE average throughput rate and spectral efficiency of the network. As shown in the graphs, we concluded that the UE average throughput keeps fixed in the range of $71-72 \mathrm{Mb} / \mathrm{s}$ for feedback delay from 1 to 6 TTI, and spectral efficiency also keeps fixed in the range of 21-21.5 bit/cu for 1-6 TTI.

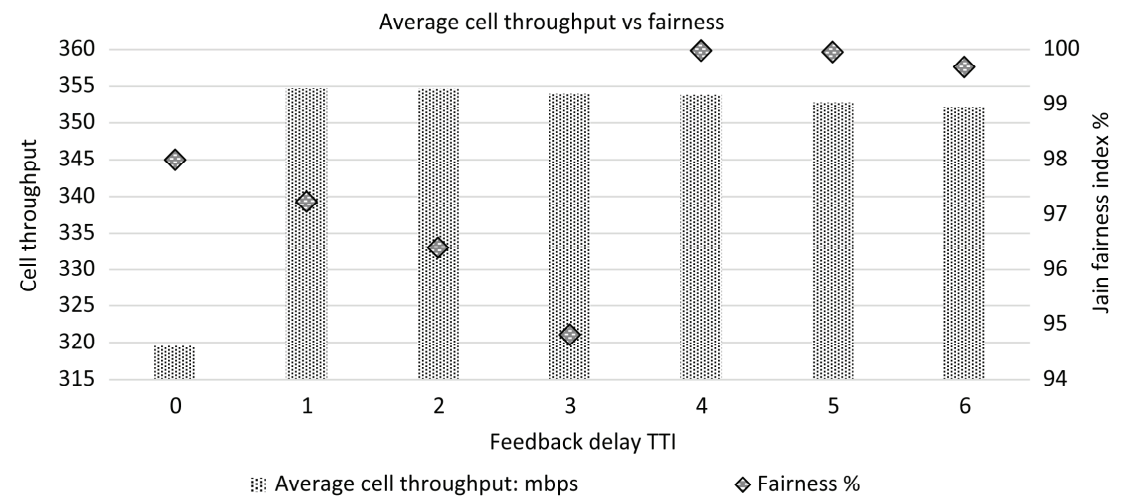

Figure 8. Performance analysis of PF scheduler in LTE-A EUTRAN with RN/RRH in term of throughput and resource fairness index. 


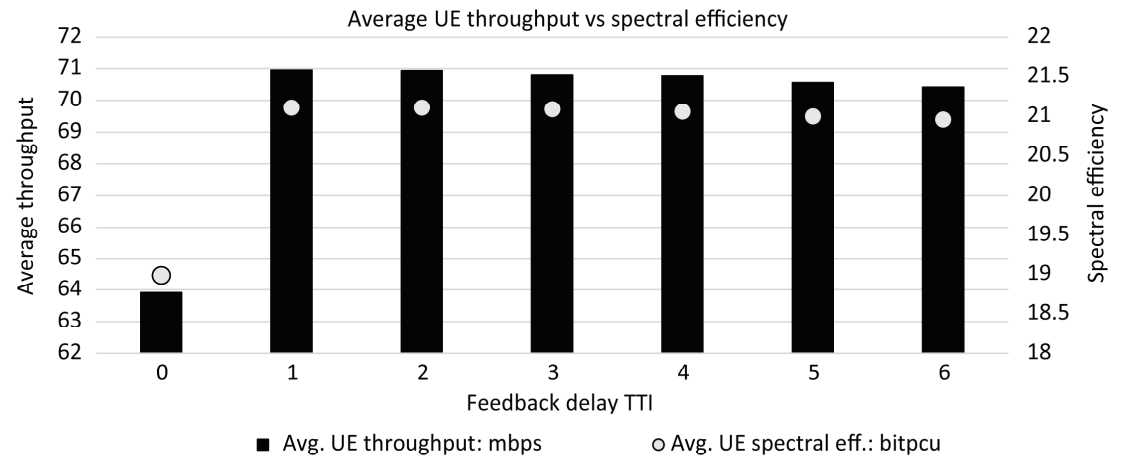

Figure 9. Performance analysis of PF scheduler in EUTRAN with RN/RRH in terms of average UE throughput and average UE spectral efficiency with varying feedback delay.
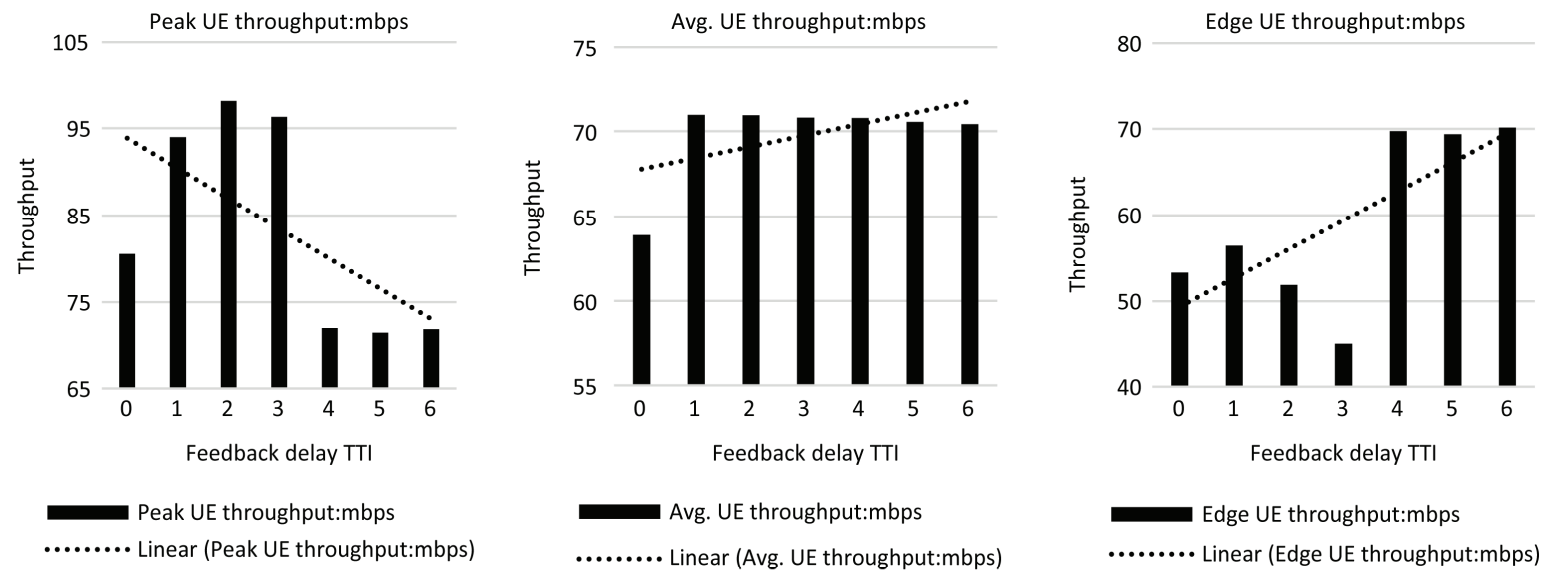

Figure 10. Throughput performance a typical PF scheduler EUTRAN network with RN/relay for peak, average, and edge UE, under varying feedback delay.

In Fig. 10, we illustrate throughput of UE with varying feedback delay for a typical PF scheduler EUTRAN network with RN/RRH. Simulated configuration is defined as per in Table 2. In the first graph, the UE peak throughput decreases when the feedback delay increases in PF scheduler. In the second graph, UE average throughput increases with the feedback delay. Similarly, in the third graph, throughput increases with the feedback delay. Here we identify a behavioral difference for UE throughput between both setups of EUTRAN LTE and EUTRAN LTE-A.

\section{Conclusion}

We have analyzed two typical EUTRAN networks with $\mathrm{PF}$ scheduler under a system level simulator with varying feedback delay (in TTI). We have identified a performance relation of different key performance indicators such as cell throughput, UE throughput, resource distribution (Jain) fairness index, and UE spectral efficiency. When comparing the effect of delay in performance of PF scheduler, we conclude that the increment in feedback delay helps to compensate the effect of an imperfect channel. The performance of LTE model improves significantly, while only a slight improvement is observed in the LTE-A model. As LTE-A is equipped with RN/RRH, the channel offers less imperfections and good channel quality. Although we observe the $\mathrm{PF}$ algorithm with $2-3$ feedback delays in TTI, better balance performance is offered in all aspects, whether it is a cell or UE throughput, RF distribution, and spectral efficiency.

\section{Conflict of Interest}

The authors declare that they have no conflict of interest.

\section{References}

[1] 3G LTE specifications [Online]. Available: http://www.3gpp. org/dynareport/36-series.htm

[2] S. Schwarz, C. Mehlfuhrer, and M. Rupp, Low complexity approximate maximum throughput scheduling for LTE, IEEE AsilomarSSC, Asilomar, 2010.

[3] A. Djouama and M.-S. Lim, Reduction of the feedback delay effect on a proportional fair scheduler in LTE downlink using nonlinear, $A E U$ - International Journal of Electronics and Communications, 69(10), October 2015, 1393-1402.

[4] R. Kwan, C. Leung, and J. Zhang, Proportional fair multiuser scheduling in LTE, IEEE Signal Processing Letters, 16(6), June 2009, 461-464.

[5] A. Saxena and R. Sindal, Performance analysis of MAC Scheduler in LTE (EUTRAN) for "ASAR": Resource allocation, Next Generation Mobile Applications, Services and Technologies (NGMAST 2016), Cardiff UK, 2016.

[6] M.T. Kawser, H.M.A.B. Farid, A.R. Hasin, A.M.J. Sadik, and I.K. Razu, Performance comparison between round robin and 
proportional fair scheduling methods for LTE, International Journal of Information and Electronics Engineering, 2(5), September 2012, 678-681.

[7] S.-B. Lee, I. Pefkianakis, A. Meyerson, S. Xu, and S. Lu, Proportional fair frequency-domain packet scheduling for $3 \mathrm{GPP}$ LTE Uplink TR-090001, UCLA CSD Technical Report.

[8] Z. Ma, W. Xiang, H. Long, and W. Wang, Proportional fair resource partition for LTE-Advanced networks with Type I relay nodes, in 2011 IEEE International Conference on Communications (ICC) (IEEE ICC), June 2011.

[9] A. Saxena and R. Sindal, Analysis of cell reselection in evolved UMTS radio access network radio resource management, 2nd IEEE International Conference on Engineering and Technology, Coimbatore, Tamil Nadu, India, 17-18 March 2016.

[10] F. Capozzi, G. Piro, L. Grieco, G. Boggia, and P. Camarda, Downlink Packet Scheduling in LTE cellular networks: Key design issues and a survey, IEEE Communications Surveys and Tutorials, 15(2), 02 May 2013, 678-700.

[11] A. Saxena and R. Sindal, Characterizing the effect of N-CoMP and CoMP with interference in LTE-A, International Conference on Computer Communication and Informatics ICCCI 2016, Coimbatore, Tamil Nadu, India, 2016.

[12] J. Li, Y.Q. Zhao, F.R. Yu, and X. Huang, Queuing analysis of two-hop relay technology in LTE/LTE - A network with unsaturated and asymmetric traffic, IEEE Internet of Things Journal, XX(YY), 2015, 1-8.

[13] C. Khirallah, D. Vukobratovi'c, and J. Thompson, Performance analysis and energy efficiency of random network coding in LTEAdvance, IEEE Transaction on Wireless Communications, 11(12), December 2012, 4275-4285.

[14] B. Guo, W. Cao, A. Tao, and D. Samardzija, LTE/LTE - A signal compression on the CPRI interface, Bell Labs Technical Journal, 18(2), 2013, 117-133.

[15] Y.B.A.I. Kostanic, Performance evaluation of proportional fairness scheduling in LTE, World Congress on Engineering and Computer Science 2013 Vol II, San Francisco, USA, 23-25 October 2013.

[16] R.K. Jain, D.-M.W. Chiu, and W.R. Hawe, A quantitative measure of fairness and discrimination for resource allocation in shared computer system, ACM Transaction on Computer Systems (DEC-TR-301), 26 September 1984, 1-36.

\section{Biographies}

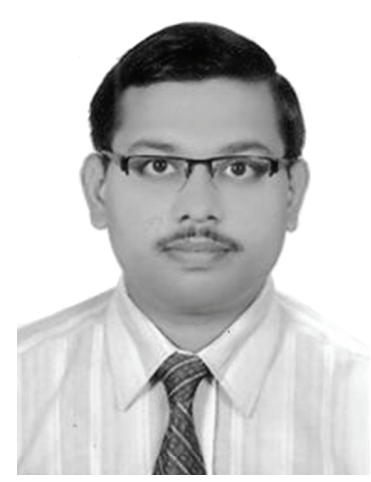

Ankit Saxena has a diploma degree in Electronic Communication. He obtained a Bachelor of Engineering degree in Electronics and Instrumentation, a Master's degree in Business Administration (System) and a Master's degree in Electronics Engineering (Electronics and Telecommunication Engineering). He started his career in the telecom industry with a major telecom operator in different technologies, Airtel in 2000, Tata (2003-2004), Reliance (2004-2008), and telecom product manufacture and managing services in Alcatel-Lucent (2008-2009) and Ericsson (2009-2015). He is currently working towards Ph.D. degree in Electronics and Telecommunication in the Laboratory of the Institute of Engineering and Technology, Devi Ahilya University, Indore. His current research is in the area of performance analysis and key performance improvement in EUTRAN network (LTE and LTE-Advance), and he is a reviewer of various international journals and conferences. E-mail: ankitsaxena4u@gmail.com

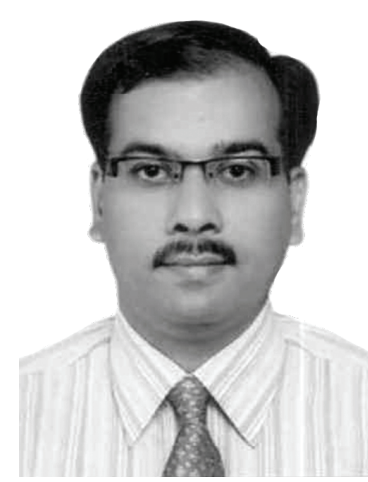

Ravi Sindal received the B.E. (Hons) degree from Rajasthan University, Jaipur in 1998. He received the M.Tech. degree in Instrumentation and Ph.D. degree in Electronics and Telecommunication from Devi Ahilya University, Indore in 2000 and 2011, respectively. Since 2013, he has been a professor of Electronics and Telecommunication at the Institute of Engineering and Technology, Devi Ahilya University, Indore. His research interests include radio resource management, modelling and simulation, digital design, and wireless networks. He has served on the programme committee of various conferences. He has published many papers in various well-known international journals and conferences. 